\title{
VERTEBROBASILAR SYSTEM IN THE EUROPEAN HARE
}

\author{
Flešárová, S., Maženský, D. \\ Department of Anatomy, Histology and Physiology \\ University of Veterinary Medicine and Pharmacy, Komenského 73, 04181 Košice \\ The Slovak Republic \\ slavka.flesarova@uvlf.sk
}

\begin{abstract}
The aim of this study was to describe the arterial arrangement of the cervical spinal cord in the hare using the corrosion technique. The study was carried out on 10 adult European hares (Lepus Europeus). The arterial system of the cervical spinal cord was injected using Batson's corrosion casting kit No. 17. The fusion of the bilateral vertebral arteries was found in $70 \%$ of the cases without a connecting branch and in $30 \%$ of the cases with one connecting branch just posterior to the fusion. The ventral spinal artery was in connection with the right vertebral artery in $60 \%$ of the cases and by means of an anastomosis of two spinal branches arising from the bilateral vertebral arteries in $40 \%$ of the cases. Based on the results of this study, it is possible to conclude that there is a high variability of the blood supply to the cervical part of the spinal cord in the hare.
\end{abstract}

Key words: basilar artery; corrosion cast; rabbit; spinal cord; ventral spinal artery

\section{INTRODUCTION}

Injuries of the cervical spinal cord usually result in full or partial tetraplegia. However, depending on the specific location and severity of the trauma, limited function may be retained for example, in the ability to breath, which makes utilization of mechanical ventilators, phrenic nerve pacing etc., unnecessary [3].

Research on the arrangement and variability of the feeding arteries of the spinal cord in several species of laboratory animals [4], [8], [9], [12] and in man [1], [5], [6] is common. The arterial arrangement of the cervical spinal cord in the European hare has not been described so far.

The vertebral artery arises from the subclavian artery and emerges from the thoracic cavity through the cranial thoracic aperture. The artery then passes through the transverse opening of the sixth cervical vertebra into the transverse canal of the cervical vertebrae, in which it continues in the cranial direction. Along its course inside the transverse canal it gives off spinal branches. These spinal branches enter the vertebral canal through the intervertebral foramina. After entering the vertebral canal they send 
ventral and dorsal branches to the spinal cord, which participate in the formation of the ventral and dorsal spinal arteries. Bilateral vertebral arteries enter the vertebral canal via lateral vertebral opening of the atlas. They fuse together on the caudal margin of the dorsal surface of the basilar part of occipital bone forming the basilar artery. The basilar artery participates in forming the arterial circle of the brain. In the place of formation of the basilar artery, it is located at the cranial connection of the spinal arteries to the vertebral arteries [4].

The aim of this study was to describe the arterial blood supply of the European hare spinal cord with a focus on the cervical region.

\section{MATERIALS AND METHODS}

This study was carried out on 10 adult European hares (Lepus europaeus, L. 1758), aged 140 days. Hares (obtained

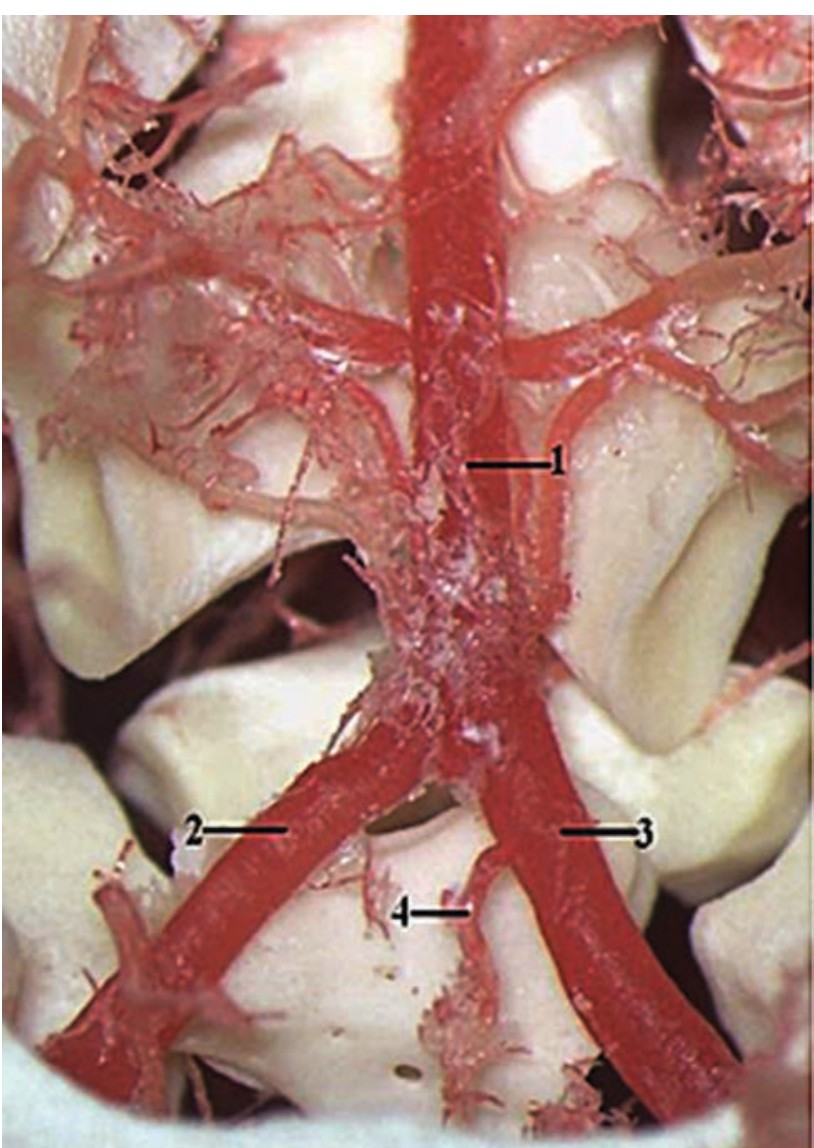

Fig. 1. Formation of the basilar artery without the posterior connec tion, the connection of the ventral spinal artery to the right vertebral artery. 1 - basilar artery; 2 - left vertebral artery; 3 - right vertebral artery; 4- ventral spinal artery. Dorsal view. Magn. $\times 12.5$ from ISFA APRC, Nitra, Slovak Republic) of both sexes (female $\mathrm{n}=5$; male $\mathrm{n}=5$ ) with weights range of $2.5-3.2 \mathrm{~kg}$ were used in an accredited experimental laboratory of the University of Veterinary Medicine and Pharmacy in Kosice. The animals were kept in cages under standard conditions (temperature $15-20^{\circ} \mathrm{C}$, relative humidity $45 \%$, 12-hour light period), and fed with a granular feed mixture (O-10 NORM TYP, Spišské kŕmne zmesi, Spišské Vlachy, Slovak Republic). The drinking water was available to all animals ad libitum. The animals were injected intravenously with heparin (50000 IU. $\mathrm{kg}^{-1}$ ) $30 \mathrm{~min}$ before they were sacrificed by the intravenous injection of embutramide (T-61;0.3 ml. $\mathrm{kg}^{-1}$ ). Immediately after euthanasia, the vascular network was perfused with a physiological solution. During manual injection through the ascending aorta, the right atrium of the heart was opened in order to lower the pressure in the vessels to ensure an optimal injection distribution. Batson's corrosion casting kit No. 17, using a volume of $50 \mathrm{ml}$ (Dione, České Budějovice, Czech Republic) was used as

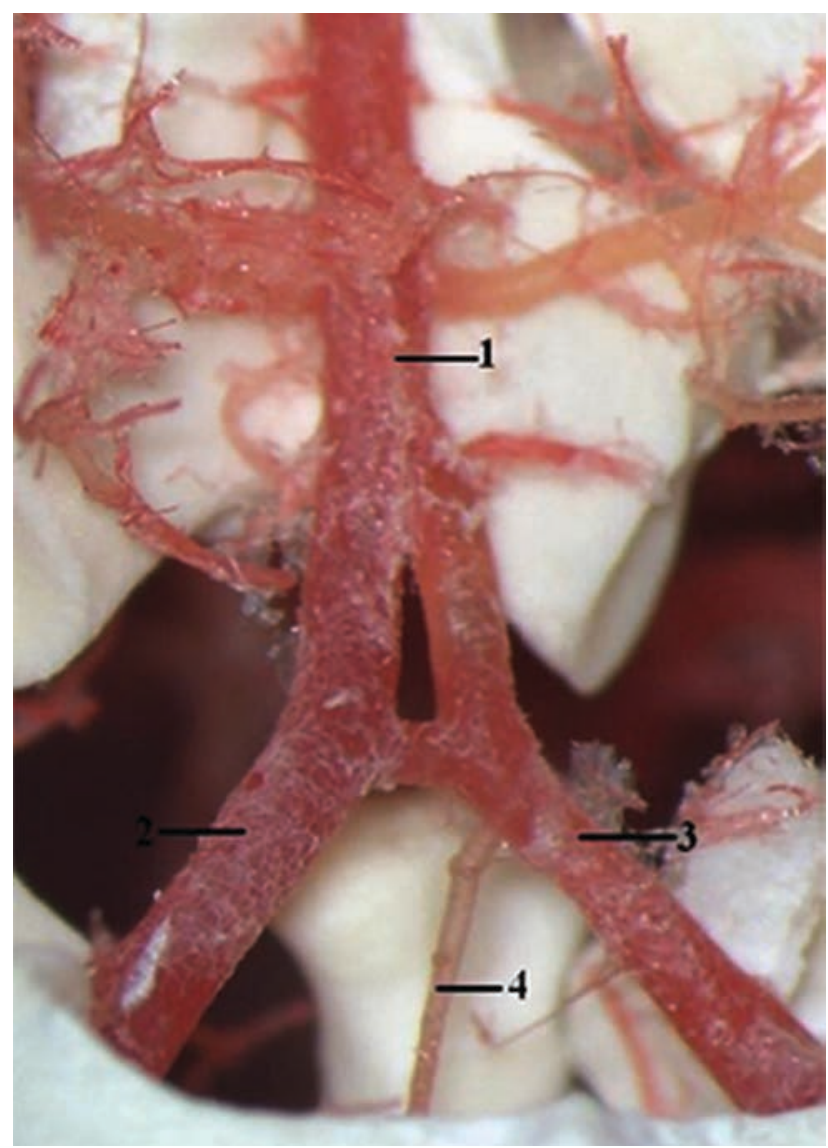

Fig. 2. Formation of the basilar artery with the posterior connection, the connection of ventral spinal artery to the right vertebral artery. 1 - basilar artery; 2 - left vertebral artery; 3 - right vertebral artery; 4 - ventral spinal artery. Dorsal view. Magn. $\times 12.5$ 


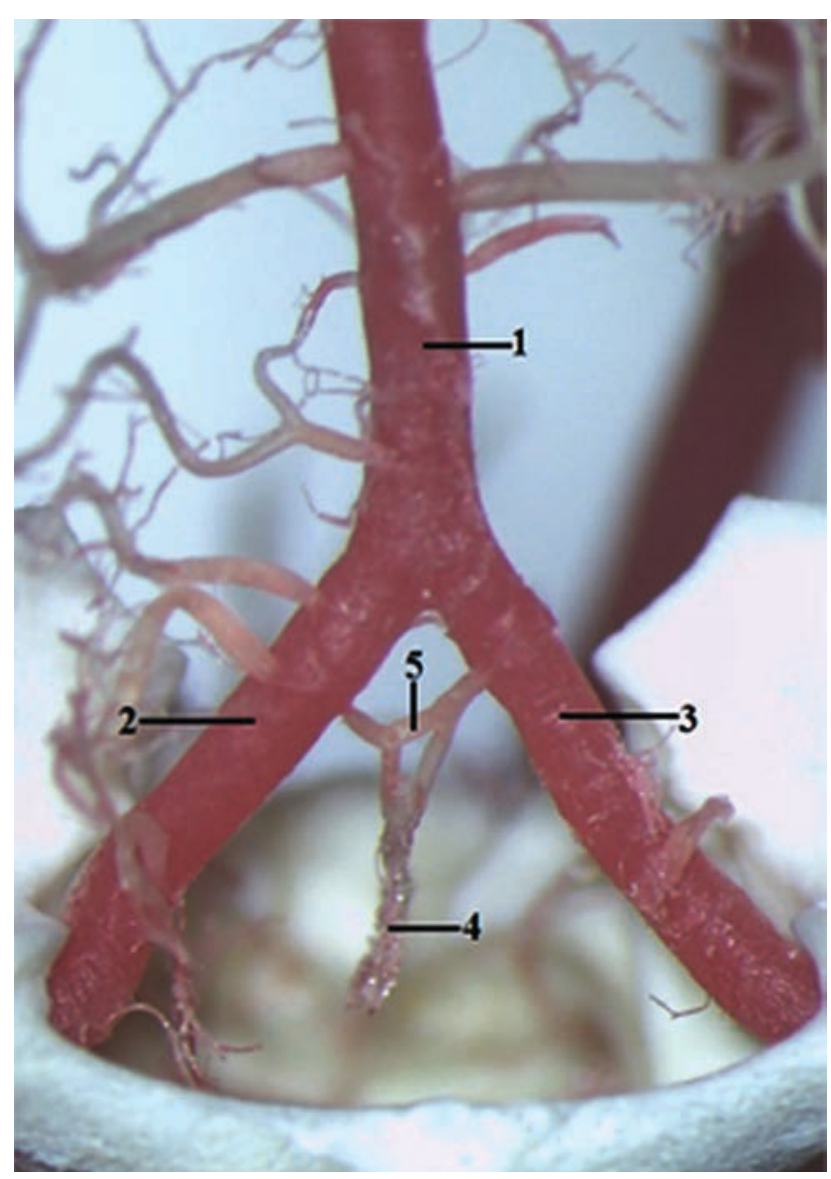

Fig. 3. Connection of the ventral spinal artery to the vertebral arteries by means of anastomosis of two spinal branches, a communicating branch between bilateral spinal branches. 1 - basilar artery; 2 - left vertebral artery; 3 - right vertebral artery; 4 - ventral spinal artery; 5 -communicating branch. Dorsal view. Magn. $\times 12.5$

the casting medium. After polymerization of the medium, maceration was carried out in $2-4 \% \mathrm{KOH}$ solution for a period of 2 days at $60-70^{\circ} \mathrm{C}$. This study was carried out under the authority of decision No. 2647/07-221/5.

\section{RESULTS}

The blood supply of the cervica 1 spinal cord is more complicated than in the other parts of the body. The vertebral artery, basilar artery and caudal cerebellar artery as branches of the basilar artery give off a lot of small branches participating in the blood supply to the most cranial part of the spinal cord.

The vertebral artery entered the vertebral canal through the foramen vertebrale laterale of the atlas. On the caudal margin of the dorsal surface of the pars basilaris ossis occipitalis it was fused with the contralateral vertebral artery.
This fusion formed the basilar artery. The fusion of the bilateral vertebral arteries was found in $70 \%$ of the cases without a posterior connection (Figs. 1, 3) and in $30 \%$ of the cases with a posterior connection (Fig. 2). At the level of the formation of the basilar artery, different connections of the ventral spinal artery to the vertebral artery were found. It was in connection with the right vertebral artery in $60 \%$ of cases (Figs. 1-2) and by means of an anastomosis of two spinal branches with the bilateral vertebral arteries in $40 \%$ of the cases. In the case of an anastomosis, a communicating branch was located above the two branches (Fig. 3). Each of these branches were in connection with the medial flank of the vertebral artery on the same side. Using this technique, it was not possible to describe the segmental arteries entering the ventral and dorsal spinal arteries.

\section{DISCUSSION}

Based on our study we can conclude that there is a different arterial arrangement in the hare compared with humans. We found the formation of the basilar artery without a posterior connection of the bilateral vertebral arteries in most cases. In humans, the basilar artery is formed by the fusion of the bilateral vertebral arteries without this connection [2]. In hares, the ventral spinal artery (in humans the anterior spinal artery) was in connection with the right vertebral artery, or by means of anastomosis of two spinal arteries originating from both vertebral arteries. These two arteries were originating from the medial flank of the vertebral artery on the corresponding sides. The anterior spinal artery in humans is formed only by the fusion of the anterior spinal branches of the vertebral arteries at the level of foramen magnum [7].

In the rabbit, the formation of the basilar artery was formed by the fusion of the bilateral vertebral arteries without any posterior connection, or two connections [4]. We found the formation of the basilar artery without any posterior connections or with one posterior connection. The origin of the ventral spinal artery was described from the right vertebral artery, from the left vertebral artery and from the anastomosis of two ventral spinal arteries, each originating from the medial flank of the vertebral artery on the same side [4]. In our study, we found the connection of the ventral spinal artery with the right vertebral artery or by means of anastomosis of two spinal branches with 
a communicating branch between each other with bilateral vertebral arteries. We did not find the connections with the dorsal spinal arteries in our study.

\section{CONCLUSIONS}

The study of the arterial patterns of the spinal cord based primarily on the use of an experimental animal might help to elucidate the principles of how the blood vessels are distributed to the spinal cord. At the same time, such an effort provides additional information concerning the manner of vascularization of the central nervous system in general [10], [11], [13].

\section{REFERENCES}

1. Alleyne, C.H., Cawley, C.M., Shengelaia, G. G., 1998: Microsurgical anatomy of the artery of Adamkiewicz and its segmental artery. J. Neurosurg., 89, 791-795.

2. Ashwini, C.H.A., Shubha, R., Jayanthi, K.S., 2008: Comparative anatomy of the circle of Willis in man, cow, sheep, goat, and pig. Neuroanatomy, 7, 54-85.

3. Cheshire, W.P., Santos, C.C., Massey, E.W., Howard, J.F., 1996: Spinal cord infarction: etiology and outcome. Neurology, 47, 321-330.

4. Mazensky, D., Danko, J., Petrovova, E., Luptakova, L., et al., 2012: Arterial arrangement of the cervical spinal cord in rabbit. Anat. Sci. Int., 87, 155-159.
5. Melissano, G., Civilini, E., Bertoglio, L., Calliari, F., Campos Moraes Amato, A., Chiesa, R., 2009: Angio-CT imaging of the spinal cord vascularisation: a pictorial essay. Eur. J. Vasc. Endovasc. Surg., 39, 436-440.

6. Nijenhuis, R. J., Leiner, T., Cornips, E. M., 2004: Spinal cord feeding arteries at MR angiography for thoracoscopic spinal surgery: feasibility study and implications for surgical approach. Radiology, 233, 541-547.

7. Shajmi, M.F., Maziak, D.E., Shajmi, F.M., Ginsberg, R. J., Pon, R., 2003: Circulation of the spinal cord: an important consideration for thoracic surgeon. Ann. Thorac. Surg., 76, 315-321.

8. Schievink, W.I., Luyendijk, W., Los, J.A., 1988: Does the artery of Adamkiewicz exist in the albino rat? J. Anat., 161, 95-101.

9. Strauch, J. T., Spielvogel, D., Lauten, A., Zhang, N., et al., 2003: Importance of extrasegmental vessels for spinal cord blood supply in a chronic porcine model. Eur. J. Cardiothorac. Surg., 24, 817-824.

10. Šulla, I., Maršala, J., Lukáč, I., 2003: A light-microscopic study of an early phase of paraplegia induced by ischemia and reperfusion in dogs. Folia Veterinaria, 47, 200-203.

11. Šulla, I., Maršala, J., Lukáč, I., 2004: A light-microscopic study of delayed phase of paraplegia induced by ischemia and reperfusion in dogs. Folia Veterinaria, 48, 76-80.

12. Tveten, L., 1976: Spinal cord vascularity IV. The spinal cord arteries in the rat. Acta Radiol., 17, 385-398.

13. Woollam, D. H. M., Millen, J. W., 1955: The arterial supply of the spinal cord and its significance. J. Neurol. Neurosurg. Psychiatry., 18, 97-102.

Received April 21, 2016 